\title{
Caracterización de los sistemas de productos en el marco de la personalización, la variabilidad y la diferenciación. Un estudio con expertos
}

\author{
Characterization of the product systems under customization, variability \\ and differentiation. A study with experts
}

John J. Cardozo V. ${ }^{1} \quad$ Bernabé Hernandis O. $^{2} \quad$ Nélida Y. Ramírez T. ${ }^{1}$

Recibido 10 de junio de 2013, aceptado 9 de enero de 2014

Received: June 10, 2013 Accepted: January 9, 2014

\begin{abstract}
RESUMEN
Desde una perspectiva sistémica, en este estudio se exploran las condiciones impuestas al diseño de sistemas de productos en contextos fundamentados en la variabilidad, la diferenciación y la personalización. Se realiza un estudio piloto para la construcción del instrumento de recolección de datos primarios. Se aplica una encuesta a 57 expertos en el área del diseño, para establecer las condiciones que deben cumplir los sistemas de productos en estos mercados. Con la aplicación de un análisis factorial exploratorio se reducen las variables del análisis; se identifican los componentes, las relaciones y las variables de diseño. Se obtienen tres componentes denominados: Capacidades, Atributos y Representación, los cuales delimitan las variables de diseño y caracterizan a los sistemas de productos. Se contrastan los hallazgos con la literatura. El artículo concluye con una discusión de las implicaciones para el diseño de sistemas de productos y las futuras investigaciones.
\end{abstract}

Palabras clave: Sistema de productos, diferenciación, variabilidad, diseño sistémico, personalización.

\begin{abstract}
From a systemic perspective, this study explores the conditions imposed on the design of systems of products in contexts grounded in variability, differentiation and customization. A pilot study for the construction of the instrument of primary data collection was performed. A survey on 57 design experts, to establish the conditions to be met by systems of products in these markets was applied. Through an exploratory factor analysis the number of variables was reduced, the components, relationships and the design variables were indentified. Three components are obtained: Abilities, Attributes and Representation; these factors demarcate the design variables and establish the character of the systems of products. Findings are contrasted with literature. The article concludes with the discussion of implications for the design of systems of products and future researches.
\end{abstract}

Keywords: Systems of products, differentiation, variability, systemic design, personalization.

\section{INTRODUCCIÓN}

Las rápidas transformaciones sociales y demográficas, resultantes de los procesos de globalización y del auge de las tecnologías que permiten intercambios de información e interacción en tiempo real, se traducen en cambios en las formas de consumo e interacción entre los usuarios y los productos, lo que obliga a los diseñadores industriales a repensar la manera cómo deben ser desarrollados los productos, replantear su rol dentro de este proceso y las implicaciones que esto pueda tener en la construcción de una sociedad,

1 Departamento de Diseño. Universidad Nacional de Colombia, Sede Palmira. Carrera 32 No 12-00 Chapinero, Vía Candelaria. Palmira, Colombia. E-mail: jjcardozov@unal.edu.co; nyramirezt@unal.edu.co

2 Departamento de Ingeniería Gráfica. Universitat Politècnica de València. Camino de Vera, s/n. C.P.: 46022. Valencia, España. E-mail: bhernand@ degi.upv.es 
cada vez más crítica e insatisfecha con la realidad en la cual está inmersa. Martins, Oliveira y Relvas [1], citando a Alves (2001), afirman que "una de las características de la globalización apoyada en el creciente aumento de la velocidad de las comunicaciones hace que las dinámicas sociales, las tendencias y las solicitudes comerciales sean cada vez más mutables".

Matulik [2] sostiene que la diferencia entre competidores locales y globales está desapareciendo lentamente; un pequeño productor local tiene consumidores globales y puede ser un buen competidor para una gran compañía ya establecida. Los consumidores tienen diferentes deseos y además la motivación y los medios para encontrar una compañía que los satisfaga, lo que, visto desde el diseño de productos se traduce en un aumento de la frecuencia de lanzamiento de productos y provoca una multiplicidad de alternativas en la oferta, de la que los consumidores seleccionan la que se ajusta con mayor precisión a sus necesidades individuales; por esto, las empresas deben ejecutar acciones innovadoras que incorporen las cualidades diferenciadoras y anticipatorias que el diseño aporta, entendiendo que la variabilidad y la personalización son ahora cualidades requeridas por los consumidores, y donde la capacidad de conceptualizar productos vinculados a la estrategia de la empresa y la dinámica del mercado es factor constructor de ventajas competitivas y de diferenciación, más ahora, que el precio ha dejado de ser el principal criterio determinante de la compra.

Se hace necesario para mantener la supervivencia de las empresas que estas asuman de lleno la dinámica del aumento en la frecuencia de introducción de productos, la reducción de tiempo y la optimización en los procesos de desarrollo, además de la necesaria concurrencia dentro de la planeación del portafolio de productos; al respecto, Erens [3] enfatiza que el advenimiento del Mercado de los Consumidores impone a las compañías satisfacer las necesidades individuales de los consumidores, pero que, sin embargo, una oferta amplia de productos no es una solución cuando va acompañada de altos costos internos.

Para Cardozo, Hernandis y Ramírez [4] la variabilidad y la personalización "exigen a las empresas una mayor complejidad en todos sus procesos, y requiere de un cambio en la manera de entender su papel en el mercado y la forma de diseñar sus productos, de modo que es necesario considerar el diseño como una capacidad que pueden desarrollar las empresas para lograr mayor competitividad". En este contexto, el enfoque de sistemas puede abordar este aumento de la complejidad mediante la modelización de las interrelaciones entre consumidores, productos y competencia dentro del mercado.

\section{MARCO DE REFERENCIA}

En este estudio se adopta el concepto "Sistema de Productos", para referirse a todos aquellos grupos de productos que son desarrollados articuladamente por una organización, con los cuales se conforma su portafolio y hacen parte de su estrategia empresarial, independientemente de las acciones, medios y lugares requeridos para su fabricación.

Con esta decisión se busca evitar la confusión en la denominación de los grupos de productos debido a que en la literatura son específicas y dependen de los enfoques disciplinares de las áreas que intervienen en la organización (marketing, finanza, producción, logística, etc.) y no atienden a criterios de uso, diseño y configuración, condiciones necesarias del contexto sociocultural y mercado actual.

A continuación se describen las características de las dinámicas de personalización, diferenciación y variabilidad, en las cuales se desenvuelven los sistemas de productos.

\section{Personalización}

Se puede interpretar el concepto de la personalización como una manera de proporcionar la satisfacción del cliente con el aumento de la variedad y la personalización de los productos, sin el correspondiente aumento en el costo y plazo de ejecución [5]; lo que, como sugiere Duray [6], "no se ajusta a la lógica tradicional de los métodos de fabricación”. En este sentido, Kotha [7], citando a Kotler (1989), afirma que el concepto de "mercado de masas" está muerto y la segmentación ha progresado hasta la era de la personalización en masa; argumenta que las empresas actualmente ya no desarrollan productos estandarizados o servicios para mercados homogéneos, y que este contexto representa la "nueva frontera" en un mundo de mercados cada vez más saturados, con lento 
crecimiento de la demanda. Silveira, Borenstein y Fogliatto [8] amplían el marco de aplicación de la personalización, al describirla como la habilidad de las empresas para producir y distribuir productos y/o servicios personalizados por medio de procesos de negocio flexibles con altos volúmenes de producción $\mathrm{y}$ con un costo razonablemente bajo.

Usui [9] establece que es posible desarrollar la personalización en cualquier etapa de la cadena de valor de un producto, lo que permite la aplicación de "niveles bajos" de personalización en las últimas etapas de esta, hasta "un alto nivel de personalización", vinculando a los consumidores desde el diseño hasta todas las etapas del desarrollo del producto.

Chen, Wang y Tseng [10] señalan que no se puede considerar equivalente la producción en masa con la personalización en masa, con la única diferencia del número de unidades producidas. Esto es debido fundamentalmente a que esta última requiere de métodos, estrategias, comportamientos y aproximaciones que vinculen más estrechamente a los consumidores con la empresa, buscando relacionar a los clientes de una manera más activa en el proceso de creación de valor de los productos.

\section{Diferenciación}

Nam [11], citando a Isaacs (1990), sostiene que la diferenciación de producto es "la distinción entre productos que cumplen el mismo propósito, pero fueron hechos por diferentes productores y, además, compiten entre ellos". Kotler [12] adopta un enfoque que abarca todos los aspectos de la oferta y afirma que diferenciación es la introducción de características diferenciales, de calidad, estilo o imagen de marca, como base para ser predominante en el mercado. Calori y Ardisson [13] afirman que estas características además deben cumplir con los siguientes requisitos: ser percibidas por los clientes, diferenciables de la imitación de los competidores y con valor para el proveedor (cuota de mercado más alta y/o mayor margen de ganancia).

Nam [11], citando a Dickson y Ginter (1987), clasifica la diferenciación de productos en física y perceptual, argumentando que cada diferenciación física es también una diferenciación perceptual; en la misma vía, Kotler [12] expone que las personas miran productos o servicios como manojos de prestaciones, y escogen la que le provee lo mejor entre las diferentes ofertas, basadas en los factores de precio y en los factores cualitativos de los productos, como desempeño, calidad, durabilidad, apariencia y estatus. Crim (1990), citado por Nam [11], afirma que la diferenciación del producto se debe enfocar en las diferencias entre los consumidores, es decir, en los usos del consumidor, la percepción de los tipos de producto y las marcas.

\section{Variabilidad}

Cardozo, Hernandis y Ramírez [4] describen cómo el diseño enfocado en la personalización y la diferenciación presenta la dificultad del aumento de la complejidad en los procesos de desarrollo, al requerir implementar la variabilidad en los productos, iniciando con el volumen de información que se genera hasta la infraestructura necesaria para la fabricación de esta variedad de productos; en esta dirección Meyer y Lehnerd [14] describen que es posible abordar y gestionar eficientemente la complejidad derivada en las familias de productos, las que tradicionalmente se definen por una matriz de segmentos del mercado que identifica grupos de usuarios particulares, y características de precio o de desempeño en los productos.

Una familia de productos puede ser entendida como una colección de variantes de productos que tienen igual o similar función, pero con diferentes combinaciones en los niveles de sus atributos. Las aplicaciones de mercado de una familia de productos, toman la forma de productos derivados basados sobre plataformas de productos. Meyer y Lehnerd [14] describen una plataforma como "un conjunto de subsistemas, componentes e interfaces que forman una estructura común, desde la que un flujo de productos derivados pueden ser eficientemente desarrollados y producidos". Zha, Sriram y Lu [15] describen cómo en el desarrollo de productos personalizados una familia de productos puede variar ampliamente la selección y el ensamble de módulos o bloques predefinidos con diferentes niveles de atracción, para así satisfacer diversos requerimientos de personalización. Chen, Wang y Tseng [10] establecen que el diseño de productos flexibles debe ser fácilmente adaptable en respuesta a las transformaciones en los requerimientos de los clientes, por medio del cambio de un pequeño número de componentes o módulos. 
Para Martins, Oliveira y Relvas [1], la concepción modular se usa principalmente en la producción de productos complejos, que pueden ser divididos en pequeños subsistemas y funcionan como un grupo de componentes interconectados. En el diseño con enfoque modular se deben abordar tres aspectos fundamentales: arquitectura de producto, que especifica los módulos y las funciones del sistema; módulos (componentes, subsistemas o mecanismos) que interactúan y ejecutan las funciones; y las interfaces que definen las conexiones y la comunicación entre los módulos. Liang y Huang [16] afirman que un desarrollo de producto modular es aquel donde el input y output de las relaciones (interfaces) entre los componentes de un producto han sido totalmente especificadas y estandarizadas.

\section{DISEÑO DE SISTEMAS DE PRODUCTOS}

\section{Interpretación sistémica del mercado}

La dinámica actual de los mercados exige a las compañías el incremento de la complejidad en todos sus procesos, y requiere un cambio en las formas de reconocer su rol y en cómo diseñar sus productos. El enfoque de sistemas permite manejar este aumento de la complejidad mediante el modelado de las interrelaciones entre los consumidores, los productos y la competencia dentro del mercado (Figura 1), interpretando a las empresas como sistemas abiertos que realizan intercambios con los otros elementos (consumidores, competencia, proveedores, etc.) del suprasistema (mercado), resultando en un mutuo intercambio y modificación de estos elementos. En contextos de acentuada diversidad, las empresas se caracterizan por un intercambio acelerado con su entorno, requiriendo lanzar productos con más frecuencia, recolectando información de los consumidores rápidamente; deben desarrollar procesos para estudiar los cambios y anticiparse a las acciones de la competencia, e incorporar e interpretar los nuevos desarrollos en materiales y procesos que presentan los proveedores.

Los límites del sistema lo diferencian de su entorno y su permeabilidad determina su capacidad para realizar intercambios. Tomando este límite como referencia, los sistemas pueden ser descritos de manera general de dos formas: externa e interna. La descripción externa vincula al sistema con el

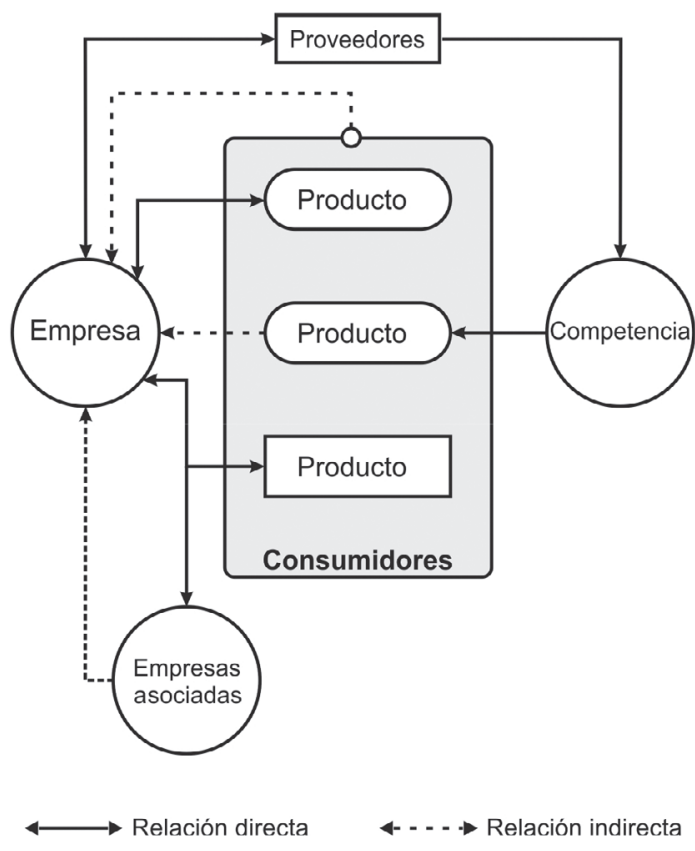

Figura 1. Interpretación sistémica del mercado.

entorno en el que opera, y lo describe en términos de entradas (inputs) y salidas (outputs).

La descripción interna de los sistemas caracteriza las relaciones entre los elementos que lo integran, y define al sistema desde un conjunto de variables y sus interacciones, en el que conceptualmente todos los sistemas existen dentro de uno mayor y por tanto pueden ser vistos a partir de su composición en subsistemas y partes (escalabilidad). Los productos establecen el vínculo de la empresa con los consumidores, pero es desde el diseño cuando se determina la apariencia (morfología), las características (funciones) y las prestaciones (usos), con las cuales se establece el tipo y la calidad de este vínculo. Hernandis y Briede [17] describen la forma cómo el diseño de nuevos productos debe responder a una serie de requerimientos y variables impuestos por otros (el mercado global, los consumidores, el departamento de producción, etc.) y una gran cantidad de información debe ser procesada cuando estos se desarrollan, manejando el proceso como una actividad simultánea e iterativa desde el principio, que incluye aspectos que fueron resueltos previamente en fases anteriores, con una estructura basada en un sistema y sus múltiples relaciones. 
Las metodologías de diseño de familias de producto de acuerdo con Simpson [18], Trott [19] y Van Wie [20] (entre otros) se centran en la determinación de las características funcionales y de producción integrando los factores morfológicos y de uso solamente en las etapas finales de desarrollo, siendo esto contradictorio, porque precisamente estos factores son los que permiten la diferenciación desde la incorporación de los criterios de los usuarios, siendo esta la base de la personalización.

Por el contrario, las aproximaciones sistémicas se basan en la modelización de los factores que integran al producto (morfología, uso y función) con el objetivo de gestionar óptimamente la información desde las fases iniciales del proceso de diseño (buscando acelerar el desarrollo de los productos), estableciendo inicialmente una definición conceptual del sistema y de las características de los elementos que lo integran, identificando los elementos comunes y definiendo las relaciones entre estos, para posteriormente extrapolar esta información al diseño de un amplio número de productos.

En el nivel de diseño de sistemas de productos se entiende que coexisten diferentes tipologías de acuerdo con las múltiples recombinaciones y diferentes grados de aplicación de productos integrantes de estos sistemas; y a su vez el diseño del sistema condiciona las características de los productos que lo componen debido a la subordinación conceptual que ejerce el sistema al subsistema; la Figura 2 ilustra estas relaciones.

\section{PLANTEAMIENTO CONCEPTUAL PARA DISEÑO DE SISTEMAS DE PRODUCTOS}

Inicialmente se detalla el proceso de diseño de productos con enfoque sistémico, para luego extrapolar las lógicas de este modelo al diseño de sistemas de producto, apoyados en la subordinación estructural y jerárquica del producto frente al sistema de productos.

\section{El modelo de diseño concurrente}

La Figura 3 representa el modelo que incorpora los criterios sistémicos en el desarrollo de productos, en el que se descompone el "problema de diseño" en elementos de menor complejidad (forma, función y ergonomía) pero manejados de modo concurrente, lo que permite la continua retroalimentación interna del sistema.

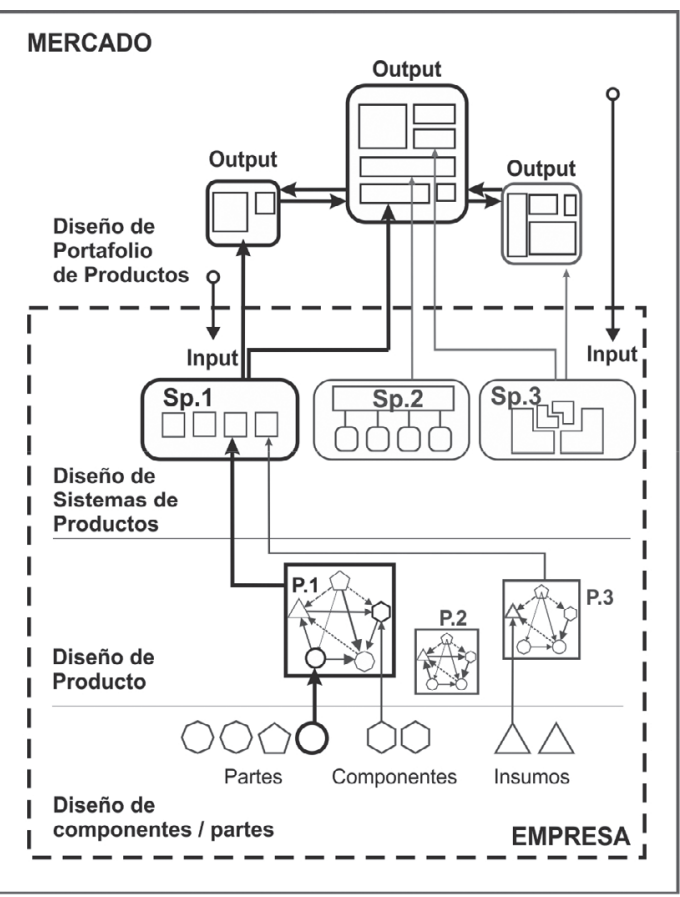

Figura 2. Descripción interna del sistema de diseño en la empresa.

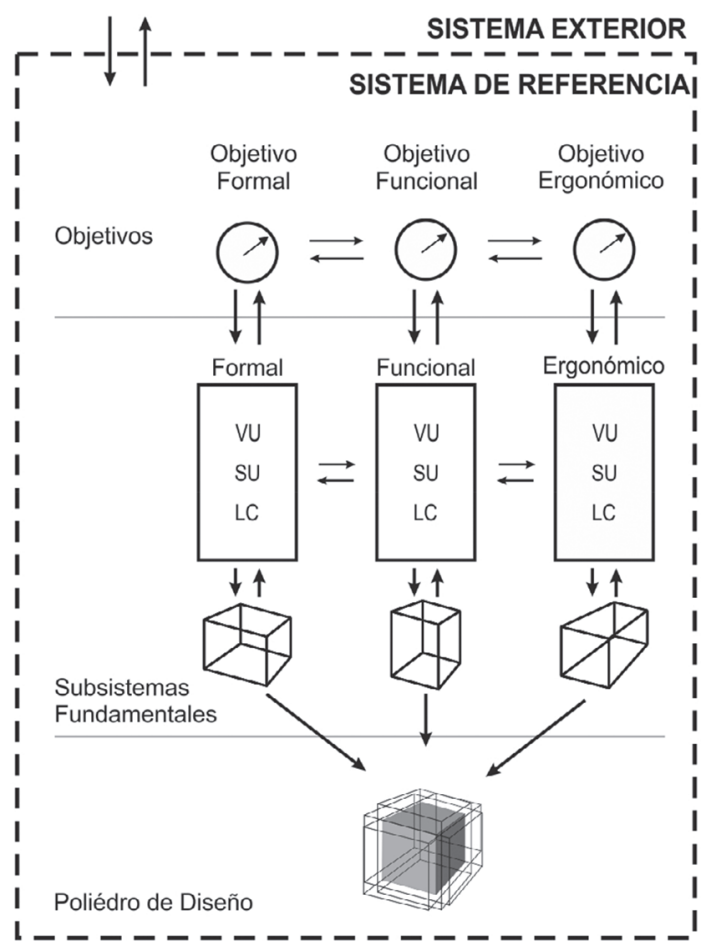

Figura 3. Modelado teórico de producto. Adaptado de Hernandis [21]. 
Siguiendo lo planteado por Hernandis [21], en el modelado del producto se establecen las condiciones, los límites, las estructuras internas y las relaciones (interfaces) que el producto debe poseer; desde el sistema exterior se incorporan las informaciones, consideraciones y condicionantes pertinentes para su configuración del producto. El sistema en estudio se subdivide para su análisis en varios sistemas o niveles fundamentales (formal, funcional y ergonómico), los cuales serán a su vez subsistemas del anterior. En el análisis del modelado teórico se establecen los elementos que componen cada uno de los subsistemas fundamentales, con el objetivo de identificar y determinar los volúmenes, superficies y límites de cada uno de estos subsistemas y sus relaciones. Posteriormente se analizan por medio de indicadores y variables para consolidar el modelado teórico. Con este análisis se hace una conformación de espacios de diseño de cada uno de los subsistemas fundamentales, los que serán integrados en el espacio conceptual del diseño, estableciendo el llamado Poliedro de Diseño [21], que define las características externas e internas que optimizan los objetivos de diseño.

\section{Modelo de diseño de sistemas de productos con enfoque sistémico}

El modelado de sistemas es análogo en su metodología al modelado de productos, variando en los criterios abordados debido al nivel jerárquico en que se encuentra dentro del sistema de diseño de la empresa. En este modelo propuesto (Figura 4) las informaciones del exterior son filtradas por los criterios de variabilidad, personalización y diferenciación.

El modelado sistémico permite realizar aproximaciones multidimensionales, para abordar la complejidad de manera ordenada y concurrente. De acuerdo con Cardozo, Hernandis y Ramírez [22], el modelado del sistema de productos está constituido por tres subsistemas, en el primero (estructura) se establece la tipología, el número de productos que la componen, la estructura de relaciones y jerarquías, las clases y la interdependencia de los productos que la integran; se prioriza el orden en que los productos serán desarrollados, estableciendo la variación del grado de complejidad de cada producto (de mayor a menor grado), para que de esta manera se puedan aprovechar las soluciones parciales que sean comunes y que paulatinamente se integren en el desarrollo de

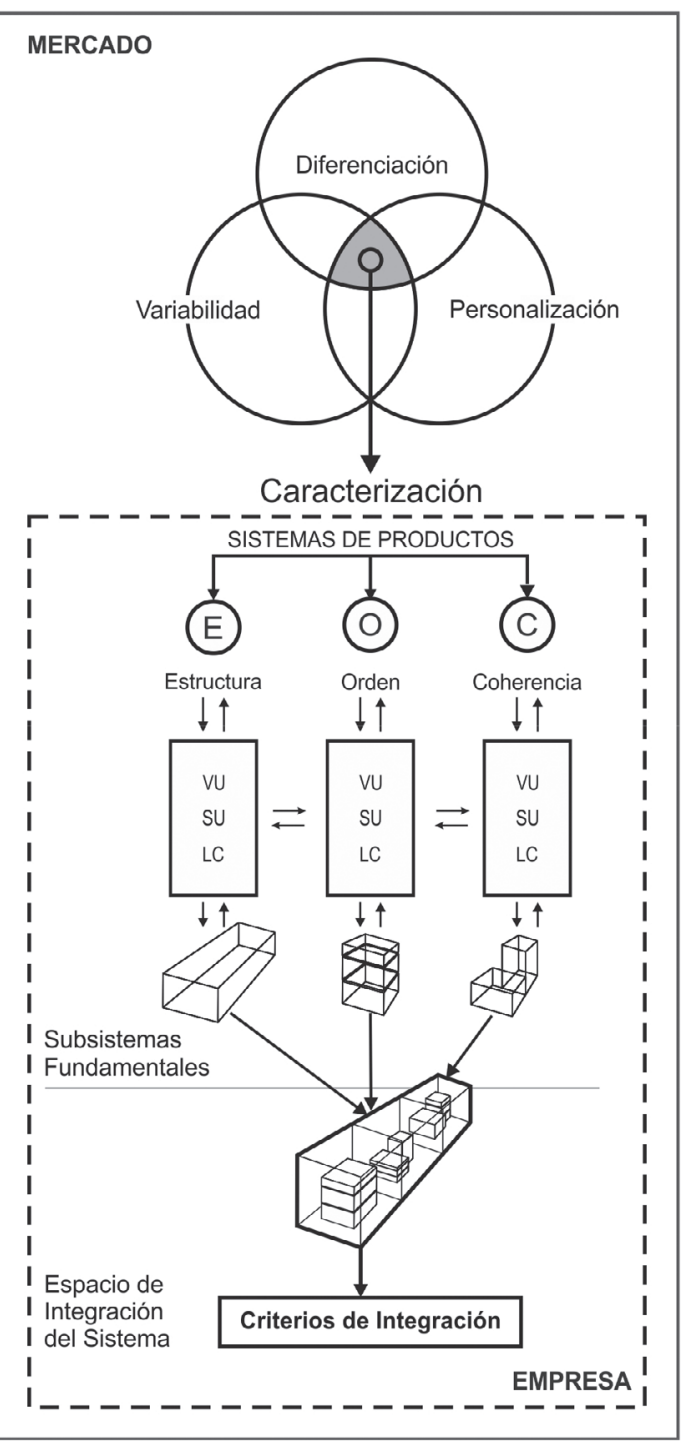

Figura 4. Modelo conceptual para el diseño de sistemas de productos.

cada producto. En el segundo subsistema (orden) se definen los subsistemas comunes y particulares entre los productos, también se establecen las relaciones intraobjetuales, y las formas y medios para su realización. En el tercer subsistema del modelado (coherencia) es donde se establecen los criterios interobjetuales vinculando los aspectos soft con los aspectos hard, también incorpora las decisiones sobre grados de complejidad en el uso y experiencia. El análisis integrado de estas dimensiones permite determinar los criterios que serán incorporados en todos los productos, identificando y caracterizando los volúmenes, superficies y límites de cada uno de 
los subsistemas y elementos que los componen. El resultado de este análisis es el espacio de integración de criterios del sistema.

\section{MODELO DE INVESTIGACIÓN}

El planteamiento de la investigación busca sustentar el modelo conceptual propuesto. El abordaje sistémico requiere de la integración de informaciones del sistema exterior, en este caso, al estar condicionado por las dinámicas de variabilidad, personalización y diferenciación, el sistema se caracteriza por la forma cómo se interpretan y filtran las informaciones de todas las audiencias del exterior, de lo anterior se desprende la hipótesis: la integración de variables enmarcadas en los criterios de variabilidad, personalización y diferenciación, caracterizan a los sistemas de productos.

\section{METODOLOGÍA}

Se diseñó una investigación descriptiva relacional cuantitativa. Las variables incorporadas son extraídas de la revisión documental y de un estudio piloto realizado con un grupo de 6 expertos académicos con experiencia en investigación, docencia y transferencia tecnológica en sus países. En el estudio piloto se discutió sobre las variables a incorporar, a partir de una selección de contenidos resultado de la revisión bibliográfica y el marco conceptual, se presentó un listado de 60 contenidos, de los cuales los especialistas seleccionaron 17 para ser incluidos en la encuesta (la Tabla 1 presenta las variables seleccionadas para el estudio).

Se determinó que las variables serían calificadas por los encuestados mediante una escala de Likert de 5 intervalos de acuerdo con lo planteado por Elejabarrieta e Íñiguez [23], la que mide el grado de importancia que tienen las variables en la caracterización de los sistemas de productos, donde 1 es el valor más bajo y 5 el más alto. Se usaron análisis estadísticos simples para probar la validez de las escalas y el cuestionario fue aplicado posteriormente [24].

\section{Muestra}

La investigación tiene un carácter teóricometodológico, y se determina aplicar la estrategia de la consulta a expertos, como sugiere Popovic [25] citando a Staszewski, ya que la "memoria del experto representa un componente general
Tabla 1. Variables del estudio.

\begin{tabular}{|l|}
\hline V1. Comparten elementos comunes. \\
\hline V2. Fabricados con igual/similar tecnología. \\
\hline V3. Elaborados con materiales iguales. \\
\hline V4. Hacen partes de la misma marca. \\
\hline V5. Colores, texturas y acabados iguales. \\
\hline V6. Enfocados en la fidelización de los consumidores. \\
\hline $\begin{array}{l}\text { V7. Enfocados en la simplificación técnica, la flexibilidad } \\
\text { operativa y la tercerización. }\end{array}$ \\
\hline V8. Amplían el ciclo de vida del producto. \\
\hline V9. Productos en múltiples segmentos de mercado. \\
\hline V10. Similitud en las características formales. \\
\hline $\begin{array}{l}\text { V11. Características funcionales comunes y/o } \\
\text { complementarias. }\end{array}$ \\
\hline V12. Comparten y/o complementan la experiencia y uso. \\
\hline $\begin{array}{l}\text { V13. Menor número de elementos vs. mayor número de } \\
\text { variantes en los productos. }\end{array}$ \\
\hline V14. Desarrollan y posicionan la imagen de marca. \\
\hline $\begin{array}{l}\text { V15. Desarrollan las capacidades y habilidades de los } \\
\text { consumidores (diferentes grados de complejidad y } \\
\text { precisión en la experiencia de uso). }\end{array}$ \\
\hline $\begin{array}{l}\text { V16. Mejoran el valor percibido de los productos (énfasis } \\
\text { en la estética y la apariencia de los productos). }\end{array}$ \\
\hline V17. Reducción de costos. \\
\hline
\end{tabular}

de conocimientos especializados en una amplia gama de habilidades cognitivas". Para establecer la población de estudio se adopta la clasificación de Liem, Abidin y Warell [26], en la que los expertos se clasifican en cuatro categorías según su nivel de experiencia y ocupación: principiante, intermedio, senior y experto.

La población de estudio son expertos académicos de universidades y centros de investigación y/o tecnológicos, quienes tienen una visión global de los procesos de desarrollo y producción con sólidos vínculos con los sectores industriales debido a los múltiples servicios de asesoría y consultoría que prestan. Por tanto es "importante", además de las características de formación, que el experto posea destrezas en investigación, consultoría y ejercicio profesional. La Tabla 2 condensa las características de los expertos consultados.

La muestra consistió de 57 expertos en diseño de 20 países, de un listado inicial constituido por 103 expertos, a quienes se envió mediante correo electrónico una solicitud de participación acompañada de los documentos de soporte y del vínculo electrónico de la encuesta. Este proceso se desarrolló en el periodo comprendido entre marzo 
y agosto de 2012; se recibieron 61 respuestas, de ellas 4 fueron no válidas, obteniendo $55,3 \%$ de respuestas positivas.

Tabla 2. Perfil de los expertos encuestados.

\begin{tabular}{|c|c|c|c|c|c|c|c|c|}
\hline & & 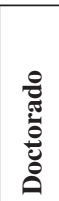 & 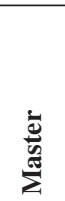 & 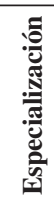 & 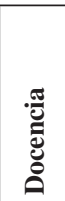 & 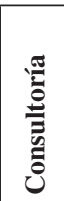 & 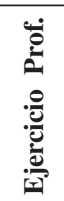 & 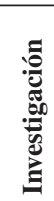 \\
\hline \multirow{2}{*}{ Experto } & & 10 & 7 & 1 & 14 & 11 & 12 & 8 \\
\hline & $\%$ & 19,2 & 13,5 & 1,9 & 26,9 & 21,2 & 23,1 & 15,4 \\
\hline \multirow{2}{*}{ Senior } & & 13 & 6 & 0 & 18 & 8 & 5 & 10 \\
\hline & $\%$ & 25,0 & 11,5 & 0,0 & 34,6 & 15,4 & 9,6 & 19,2 \\
\hline \multirow{2}{*}{ Intermedio } & & 7 & 2 & 0 & 9 & 6 & 6 & 3 \\
\hline & $\%$ & 13,5 & 3,8 & 0,0 & 17,3 & 11,5 & 11,5 & 5,8 \\
\hline \multirow{2}{*}{ Principiante } & & 1 & 5 & 0 & 3 & 1 & 2 & 4 \\
\hline & $\%$ & 1,9 & 9,6 & 0,0 & 5,8 & 1,9 & 3,8 & 7,7 \\
\hline \multirow{2}{*}{ Total } & & 31 & 20 & 1 & 44 & 26 & 25 & 25 \\
\hline & $\%$ & 59,6 & 38,5 & 1,9 & 84,6 & 50,0 & 48,1 & 48,1 \\
\hline
\end{tabular}

\section{ANÁLISIS DE DATOS}

En primera instancia se realizaron análisis de confiabilidad para medir la consistencia interna de los datos. Posteriormente se aplicó un análisis factorial (AF) con el objetivo de simplificar la información obtenida de las correlaciones entre las variables y permitir una mejor interpretación; complementariamente se estudió la frecuencia y la media aritmética de las repuestas en el conjunto de variables. En los análisis de datos se utilizó el software IBM SPSS Statistics, V20.

\section{Consistencia interna}

Se utilizó el cálculo del coeficiente $\alpha$ de Cronbach para evaluar la fiabilidad de la escala; el valor encontrado resultado del análisis fue: $\alpha=0,819$; este resultado es considerado óptimo, teniendo en cuenta lo afirmado por Oviedo y Campo [27], donde el valor mínimo aceptable para el coeficiente alfa de Cronbach es 0,70; por debajo de ese valor la consistencia interna de la escala utilizada es baja. Desde el punto de vista de la validez de contenido, en el estudio se adoptaron escalas de la revisión de la literatura; las fuentes adaptadas para analizar los contenidos de Variabilidad, Personalización y Diferenciación se encuentran en: Dell'Era y Verganti [28], Guo [29]; Candi y Saemundsson [30]; Jiao y Tseng [31]; Agard [32]; Simpson, Siddique y Jiao [33]; Hölttä-Otto [34]; Lau, Yam y Tang [35]; Marshal [36]; Sun, Sridhar y O’Brien [37] y Cormier [38].

\section{Análisis factorial (AF)}

Para el estudio de las relaciones y asociaciones entre las variables se aplicó un análisis factorial exploratorio con rotación Varimax con Kaiser (Tabla 3). Se observa que el KMO obtenido $(0,646)$ está dentro del límite de los valores recomendados por Kaiser, Meyer y Olkin (nivel aceptable).

El Análisis factorial exploratorio permitió reducir las variables originales a tres componentes explicando el $56,34 \%$ de la varianza de los datos originales ( $\mathrm{f} 1=22,78 \%$; $\mathrm{f} 2=18,2 \%$ y $\mathrm{f} 3=15,35 \%$ ), siendo este un buen resultado, tal como sugiere Macía [39], ya que en general se consideran porcentajes de varianza explicada adecuados sobre el $30 \%$. Se descartan del análisis las variables V1 y V9, debido a que sus cargas factoriales fueron inferiores a 0,4 .

Tabla 3. KMO y cargas factoriales.

\begin{tabular}{|c|c|c|}
\hline \multicolumn{2}{|c|}{$\begin{array}{l}\text { KMO Medida de adecuación muestral de } \\
\text { Kaiser-Meyer-Olkin. }\end{array}$} & 0,646 \\
\hline \multirow{3}{*}{$\begin{array}{l}\text { Prueba de } \\
\text { esfericidad } \\
\text { de Bartlett }\end{array}$} & $\begin{array}{l}\text { Chi-cuadrado } \\
\text { aproximado }\end{array}$ & 313,702 \\
\hline & gl. & 105 \\
\hline & Sig. & 0,000 \\
\hline \multicolumn{2}{|c|}{ Varianza total explicada } & $56,34 \%$ \\
\hline \multicolumn{2}{|c|}{ \% Varianza COMPONENTE 1: } & $22,78 \%$ \\
\hline \multicolumn{2}{|c|}{ V17. Reducen el costo final. } & 0,795 \\
\hline \multicolumn{2}{|c|}{ V8. Amplían ciclo de vida. } & 0,791 \\
\hline \multicolumn{2}{|c|}{ V13. Menor número de elementos. } & 0,756 \\
\hline \multicolumn{2}{|c|}{ V7. Simplificación técnica. } & 0,674 \\
\hline \multicolumn{2}{|c|}{ V12. Complementan experiencia y uso. } & 0,638 \\
\hline \multicolumn{2}{|c|}{ V15. Diferentes grados de complejidad. } & 0,537 \\
\hline \multicolumn{2}{|c|}{ V11. Características funcionales similares. } & 0,465 \\
\hline \multicolumn{2}{|c|}{ \% Varianza COMPONENTE 2 : } & $18,20 \%$ \\
\hline \multicolumn{2}{|c|}{ V2. Tienen igual tecnología. } & 0,849 \\
\hline \multicolumn{2}{|c|}{ V3. Tienen materiales iguales. } & 0,806 \\
\hline \multicolumn{2}{|c|}{ V5. Colores, texturas y acabados iguales. } & 0,670 \\
\hline \multicolumn{2}{|c|}{ V10. Características formales similares. } & 0,492 \\
\hline \multicolumn{2}{|c|}{ V6. Fidelización de los consumidores. } & 0,468 \\
\hline \multicolumn{2}{|c|}{ \% Varianza COMPONENTE 3: } & $15,35 \%$ \\
\hline \multicolumn{2}{|c|}{ V14. Posicionamiento de imagen de marca. } & 0,815 \\
\hline \multicolumn{2}{|c|}{ V16. Mejoran el valor percibido. } & 0,750 \\
\hline \multicolumn{2}{|c|}{ V4. Hacen parte de la misma marca. } & 0,620 \\
\hline \multicolumn{3}{|c|}{$\begin{array}{l}\text { Método de extracción: Análisis de componentes } \\
\text { principales. Método de rotación: Normalización Varimax } \\
\text { con Kaiser. } \\
\text { a. La rotación ha convergido en } 8 \text { iteraciones }\end{array}$} \\
\hline
\end{tabular}


La metodología del AF requiere de la identificación de las variables de cada componente y la posterior asignación de un término que denomine este conjunto de variables; por tanto en el componente 1 se encuentran ubicadas las variables V7, V8, V11, V12, V13, V15 y V17, que representan las capacidades de los sistemas de productos; el componente 2 se compone con las variables V2, V3, V5, V6 y V10, e integra los atributos que son especialmente reconocidos por los consumidores; en el componente 3 las variables V4, V14 y V16 se relacionan con los aspectos estratégicos, de posicionamiento y de representación de la empresa.

\section{RESULTADOS}

En el componente Capacidades $(22,78 \%$ de la varianza) se obtuvieron saturaciones o cargas factoriales positivas y altas en los ítems V17, V8 y V13. Esto indica que los sistemas de productos se caracterizan a partir de los criterios de personalización, pero establece que es fundamental incorporar criterios de diferenciación enfocados a potenciar el uso y las experiencias de los consumidores (V12 y V15). El componente Atributos (18,20\% de la varianza) contiene indicadores positivos altos en V2 y V3, propios de la variabilidad como criterio posibilitador de la personalización; dos ítems (V10 y V6) con cargas factoriales medias completan este factor, y sugieren la necesidad de interpretar desde la variabilidad aspectos perceptuales sensibles para los consumidores. En el tercer componente o de Representación (15,35\% de la varianza) se concentran las variables que potencian la diferenciación (V14, V16 y V4). Se resalta el papel determinante que desempeñan los sistemas de productos en la configuración y desarrollo de los portafolios de las empresas y en el logro de los objetivos estratégicos de la organización.

Lo anterior se refuerza a partir del análisis de la representación gráfica de los resultados obtenidos (Figura 5), el que, como sugieren Salvador y Gargallo [40], se realiza tomando pares de componentes, donde cada uno representa un eje de coordenadas (ejes factoriales); las coordenadas de las variables vienen dadas por sus correspondientes coeficientes de correlación para cada factor, y permiten descubrir la estructura latente del factor.
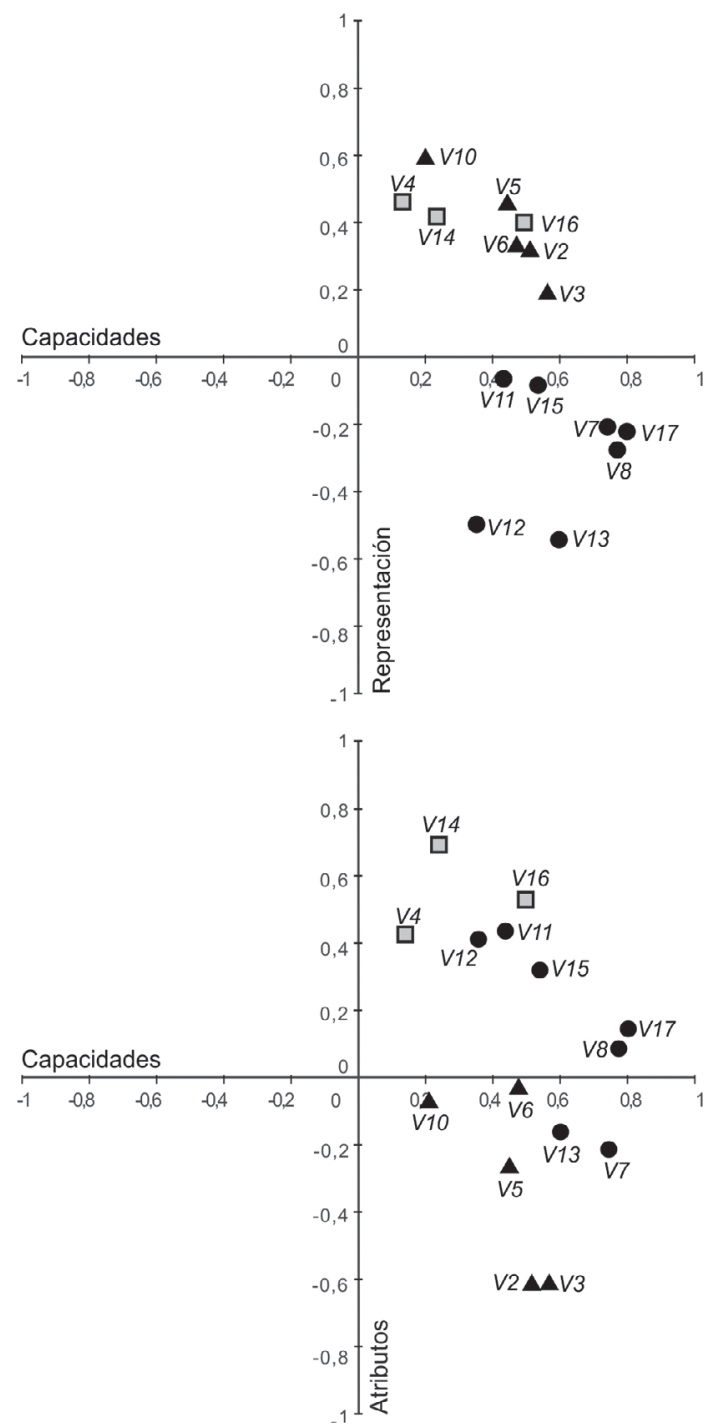

Figura 5. Representación gráfica mediante ejes factoriales.

El componente Capacidades (eje de coordenadas X) es definido por las variables V17, V8, y V7, ya que incorporan las características de la personalización por su relación con la ampliación del ciclo de vida y reducción de los costos de producto, mediante la simplificación técnica. El segundo componente o de los Atributos (eje de coordenadas Z), las variables V4 y V14 lo definen, determinando como muy importantes los criterios de marca; además existe una marcada influencia de la variable V10, que refuerza la idea de vincular los aspectos de coherencia formal (inter-intra Figura 1). En 
el componente de la Representación (eje de coordenadas Y) no hay variables que determinen específicamente su enfoque, sino por el contrario, las variables en su conjunto influencian el enfoque de los otros componentes.

Complementariamente y a manera de contraste de resultados se realizó un análisis de la media aritmética y de la frecuencia de las respuestas de los expertos en las 17 variables estudiadas (ver Figura 6); este universo muestral se analizó en una escala de 1 a 5 (donde 1 es nada importante y 5 muy importante); se aprecia de manera general que entre los expertos existe una valoración positiva (muy importante y más importante) para el $82 \%$ de las variables.

De manera específica, se observa que los valores de media más altos (V12, V14, V6, V4, V11, V16, V15) corresponden a variables enfocadas directamente en la diferenciación y en los atributos que mejoran y potencian la relación del consumidor con el sistema de productos. Es importante resaltar que la variable V1, que hace parte del criterio variabilidad, también recibió una evaluación alta. En la gráfica de frecuencias de la Figura 6 se puede reconocer que las variables asociadas con la diferenciación enfocadas en el uso y la experiencia son las que recibieron una mayor valoración por parte de los expertos (superior al $40 \%$ de valoración "muy importante"), en particular las variables V14, V12, V4 y V6.

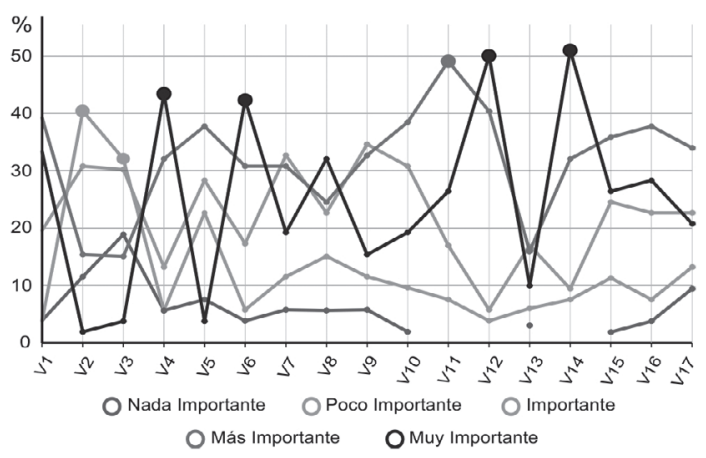

Figura 6. Gráfico de frecuencias.

Las variables con menor valoración en su grado de importancia para la caracterización de los sistemas de producto son aquellas que están directamente relacionadas con los criterios de variabilidad desde la perspectiva de la técnica (V2, V3, V13); en contraste, las variables V1 y V11, que también hacen parte de este criterio, recibieron una valoración superior. Las variables relacionadas con la diferenciación y la personalización (V5, V7, V8, V9, V10, V15, V16, V17), en su conjunto, recibieron valoraciones medias y media alta, lo que sugiere que para los expertos estos criterios son importantes y son la base de la competitividad en los mercados actuales. La variable V13 (menor número de elementos) recibió la valoración más baja del conjunto de variables, siendo esto significativo porque este criterio es la base de la variabilidad; en este caso esta valoración se interpretó de una manera más amplia y sistémica, y en el estudio se consideran estos criterios desde el enfoque del compartir elementos comunes y la perspectiva de los intercambios (protocolos, mensajes, usos, valores, experiencias, etc.) de los productos entre sí, con los consumidores y con el mercado.

\section{DISCUSIÓN}

Este estudio identifica nuevas ideas y relaciones complementarias a la literatura existente, las cuales se discuten en relación con los conceptos generales desarrollados: la variabilidad, personalización y diferenciación como caracterizadores de los sistemas de productos.

Los hallazgos de este estudio exploratorio permiten afirmar que los sistemas de producto están determinados por los usos y experiencias que desarrollen "para" y "con" los consumidores, es decir, los criterios de diferenciación orientan el carácter de estos sistemas; es importante resaltar cómo la variabilidad "desde la opinión de los expertos" se constituye en un posibilitador y no en la base para el desarrollo de las capacidades de los sistemas de producto. Lo anterior contrasta con los planteamientos de Meyer y Lehnerd [14] en la medida en que el origen del sistema no son los componentes, sino las capacidades requeridas por los usuarios; también contrasta con los planteamientos de Ulrich y Tung [41], porque las acciones de diseño y conceptualización parten del usuario y las interacciones que este pueda tener con otros usuarios y productos, y no con la integración de elementos más pequeños.

Otro aspecto relevante se encuentra en el cómo son concebidos la infraestructura y los medios de fabricación en el proceso desarrollo de los 
productos. En ese sentido Simpson, Siddique y Jiao [33] proponen la plataforma de productos como un medio para la reducción de costes y el aumento la flexibilidad de manera alternativa. En este estudio se interpretan como posibilitadores de las estrategias de la organización, fundamentadas en la homogenización, tercerización y deslocalización de las tecnologías y los medios de fabricación resultantes del auge de las TIC.

El uso y la experiencia como factor determinante difiere de los estudios de Zha, Sriram y Lu [15], en el sentido en que el sistema, más que una colección de variantes de producto, es un conjunto de experiencias en diferentes grados de intensidades. Adicionalmente se encuentra gran coincidencia con los planteamientos de Crim [42], en el sentido en que la diferenciación del producto se debe enfocar en la diferenciación de los consumidores; es decir, en establecer grados o niveles en los cuales los diferentes grupos de consumidores puedan encontrar un espacio de interacción con los productos del sistema. En este mismo sentido, los hallazgos nos muestran un alto énfasis en los atributos perceptuales en los productos, coincidiendo con los planteamientos de Agard [43] y Johansson [44], en los que la diferenciación se consigue mediante atributos que resalten las diferencias perceptuales. Además, los hallazgos indican que existe relación entre el modelo propuesto y los planteamientos de Otto y Wood [45], y que la escalabilidad es una propiedad que caracteriza estos conjuntos, donde la estructura determina la correspondencia entre los elementos (módulos) y subconjuntos.

También se ha encontrado coincidencia con los planteamientos de Bloch [46] y Lee [47], para quienes la interacción entre forma y función mediante la incorporación de características hedónicas y utilitarias explican cómo adicionar valor al producto mediante el incremento del número de experiencias asociadas al uso del producto.

Se encontró disociación entre los resultados del análisis factorial y el análisis de la frecuencia de las respuestas de los expertos en las variables V2 y V3, ya que estas tienen una alta carga factorial y bajo valor en la frecuencia. Esto sugiere que en el componente Capacidades estas variables se deben observar a través de estudiar la forma cómo potencian las experiencias y el uso en los consumidores, aportando diferentes grados de complejidad y evitando generar desfases en las interacciones entre los productos del sistema y sus múltiples usuarios, lo que se logra manejando e implementando tecnologías complementarias entre sí.

\section{CONCLUSIONES}

Se puede afirmar que la hipótesis se confirma: los análisis implementados permiten identificar los componentes que caracterizan a los sistemas de producto desde la variabilidad, personalización y diferenciación.

De manera consistente con la literatura, Luo y Chang [48], Tseng y Jiao [5], a partir de la opinión de los expertos, este estudio identifica las variables, las características y las condiciones que deben ser incorporadas en el desarrollo de sistemas de producto.

Se identifican tres componentes: el primero concentra los criterios de diferenciación, el segundo los de variabilidad y el tercero amplía las fronteras de la personalización. Se reinterpreta la influencia de los criterios de fabricación debido al peso que la deslocalización y la tercerización ejercen en el proceso desarrollo de productos para mercados de notable competitividad, trasladando esta influencia al desarrollo de la marca mediante el desarrollo de portafolios de productos enfocados al consumidor.

Sin embargo y contrariamente a la literatura existente, la variabilidad en este estudio se interpreta como un posibilitador, como la capacidad que tienen las organizaciones para disponer de la mejor oferta de productos ajustada a las necesidades de los consumidores, y no solamente como una estrategia de fabricación para la reducción de costos y tiempos de entrega.

Se denota una clara preferencia de los expertos hacia los criterios de diferenciación mediante el desarrollo de las capacidades y habilidades de los consumidores, ofreciendo productos con diferentes grados de complejidad y precisión en la experiencia de uso, lo que se desmarca de los planteamientos generales relacionados con el diseño de familias de productos, plataformas y modularidad, principalmente en cuál fase del proceso desarrollo de productos estos criterios deben implementarse. 
Un aspecto importante a resaltar se encuentra en el origen del proceso de diseño, el que de acuerdo a la opinión de los expertos se halla en la determinación del uso y las experiencias que el usuario pueda encontrar y desarrollar con los productos; en contraste con los planteamientos en los cuales se requiere inicialmente identificar las funciones y la posterior materialización de estas en elementos físicos, que responde a la lógica de la racionalidad técnica.

Este estudio exploratorio tuvo una serie de limitaciones, las que han permitido identificar las líneas de los futuros estudios. Inicialmente, el enfoque teórico conceptual sugiere que los planteamientos obtenidos se contrasten con casos del mercado y la industria. También es importante estudiar cómo los componentes identificados (capacidades, atributos y representación) se integran en los sistemas de productos, a cuáles subsistemas pertenecen y cómo se desarrollan sus interrelaciones. De forma complementaria, se debe evaluar si estas dimensiones y las variables asociadas tienen un comportamiento regular cuando se determinan diferentes configuraciones (categorías y/o tipologías) en los sistemas de productos.

\section{REFERENCIAS}

[1] J.C. M. Martins, J.A. Oliveira and C.M. Relvas. "Aesthetic, functional and manufacturing issues in the design of modular products". International Conference on Design Research - Joining Forces. Helsinki, Finland. September 22-24, 2005.

[2] P. Matulík. "Mass Customization". Doctoral Thesis. Tomas Bata University. Zlin, República Checa. 2008.

[3] F. Erens. "The Synthesis of Variety : Developing Product Families". Doctoral Thesis, Eindhoven University of Technology. Eindhoven, Netherlands. 1996.

[4] J.J. Cardozo, B. Hernandis y N. Ramírez. "El diseño de productos en el contexto de la Personalización en Masa". Revista Iconofacto. Vol. $9 \mathrm{~N}^{\circ} 12$, pp. 136-153. Enero-Junio. 2013. ISSN: 1900-2785.

[5] M.M. Tseng and J. Jiao. "Design for Mass Customization". CIRP Annals Manufacturing Technology. Vol. 45, Issue 1, pp. 153-156. 1996. ISSN: 0007-8506.
[6] R. Duray, P. Ward, G. Milligan and W. Berry. "Approaches to mass customization: configurations and empirical validation". Journal of Operations Management. Vol. 18, Issue 6, pp. 605-625. 2000. ISSN: 0272-6963.

[7] S. Kotha. "From mass production to mass customization: The Case of the National Industrial Bicycle Company of Japan”. European Management Journal. Vol. 14, Issue 5, pp. 442-450. 1996. ISSN: 1474-6085.

[8] G. Silveira, D. Borenstein and F. Fogliatto. "Mass Customization: Literature Review and Research Directions". International Journal of Production Economics. Vol. 72, Issue 1, pp. 1-13. 2001. ISSN: 0925-5273.

[9] T. Usui. "Mass Customization: Levels of Customization and Operational Capabilities". Journal of Commerce. Vol. 2, Issue 20, pp. 315-331. 2004. ISSN: 1340-914X. DOI: 10291/8564.

[10] S. Chen, Y. Wang and M. Tseng. "Mass customization as a collaborative engineering effort". International Journal of Collaborative Engineering. Vol. 1, Issue 1, pp. 152-167. 2009. ISSN: 1745-0039. DOI: IJCE.2009.027444.

[11] K.Y. Nam. "Industrial Design Strategies for Product Differentiation". Doctoral Thesis. Manchester Metropolitan University. Manchester, UK. 2011.

[12] P. Kotler. "From Mass Marketing to Mass Customization". Strategy \& Leadership. Vol. 17, Issue 5, pp. 10-47. 1989. ISSN: 1087-8572. DOI: 10.1108/eb054267.

[13] R. Calori and J.M. Ardisson. "Differentiation strategies in 'stalemate industries". Strategic Management Journal. Vol. 9, Issue 3, pp. 255 296. May/June, 1988. ISSN: 1097-0266. DOI: $10.1002 / \mathrm{smj} .4250090305$.

[14] M.H. Meyer and A.P. Lehnerd. "The Power of Product Platforms". Free Press. New York, USA. 1997. ISBN: 0-684-82580-5.

[15] X.F. Zha, R.D. Sriram and W.F. Lu. "Evaluation and Selection in Product Design for Mass Customization: A Knowledge Decision Support Approach". Artificial Intelligence for Engineering Design. Vol. 18, Issue 1, pp. 87-109. 2004. ISSN: 0890-0604. DOI: $10.1017 / \mathrm{S} 0890060404040077$.

[16] W. Liang and C. Huang. "The agent-based collaboration information system of product development". International Journal of 
Information Management. Vol. 22, Issue 3, pp. 211-224. 2002. ISSN: 0268-4012. DOI: 10.1016/S0268-4012(02)00006-3.

[17] B. Hernandis O. and J. Briede W. "An educational application for a product design and engineering systems using integrated conceptual models". Ingeniare. Revista chilena de ingeniería. Vol. $17 \mathrm{~N}^{\circ} 3$, pp. 432442. 2009. ISSN: 0718-3305.

[18] T. Simpson. "A Concept Exploration Method for Product Family Design". Doctoral Thesis. Georgia Institute of Technology. Georgia, USA. 1998.

[19] P. Trott. "Innovation Management and New Product Development". Third Edition. University of Portsmouth Business School, Pearson Education Limited. Harlow, England. 2005. ISBN: 0-273-68643-7.

[20] M. Van Wie. "Designing product architecture: a systematic method". Doctoral Thesis. University of Texas. Austin, USA. 2002.

[21] B. Hernandis O. "Desarrollo de una metodología sistémica para el diseño de productos industriales". Tesis para optar al grado de doctor. Universitat Politècnica de València. Valencia, España. 2003.

[22] J. Cardozo, B. Hernandis and N. Ramírez. "The Systemic Perspective in Conceptual Product Design in the Context of Strong Diversity". The International Journal of Design Management and Professional Practice. Vol. 6, Issue 4, pp. 39-56. 2013. ISSN: 2325-162X.

[23] F.J. Elejabarrieta y L. Iñiguez, "Construcción de Escalas de Actitud Tipo THURST y LIKERT". La Sociología en sus escenarios. $N^{\circ}$ 17. 2008. ISSN: 0123-8973.

[24] B. Flynn, S. Sakakibara, R. Schroeder and K. Bates. "Empirical research methods in operations management". Journal of Operations Management. Vol. 9, Issue 2, pp. 250-285. 1997. ISSN: 0272-6963.

[25] V. Popovic. "Expertise development in product design-strategic and domain-specific knowledge connections". Design Studies. Vol. 25, Issue 5, pp. 527-545. September, 2004. ISSN: 0142-694X. DOI: 10.1016/j. destud.2004.05.006

[26] A. Liem, S. Abidin and A. Warell. "Designers' perceptions of typical characteristics of form treatment in automobile styling". 5th International Workshop on Design \& Semantics of Form \& Movement. Taipei, China. October 26-27, 2009.

[27] H.C. Oviedo and A. Campo A. "Aproximación al uso del coeficiente alfa de Cronbach". Revista colombiana de psiquiatría. Vol. 34 $\mathrm{N}^{\circ}$ 4, pp. 572-580. 2005. ISSN: 0034-7450.

[28] C. Dell'Era and R. Verganti. "Strategies of Innovation and Imitation of Product Languages". Journal of Product Innovation Management. Vol. 24, Issue 6, pp. 580599. November, 2007. ISSN: $1540-5885$. DOI: $10.1111 / \mathrm{j} .1540-5885.2007 .00273 . \mathrm{x}$

[29] L. Guo. "Product Design and Financial Performance". Design Management Journal, Vol. 5, Issue 1, pp. 5-19. October, 2010. ISSN: 1948-7177. DOI: $10.1111 /$ j.1948-7177.2010.00010.x

[30] M. Candi and R.J. Saemundsson. "Exploring the Relationship Between Aesthetic Design as an Element of New Service Development and Performance". Journal of Product Innovation. Vol. 28, Issue 4, pp. 536-557. July, 2011. ISSN: 1540-5885. DOI: $10.1111 / \mathrm{j} .1540-5885.2011 .00827 . \mathrm{x}$

[31] J. Jiao and M.M. Tseng. "Fundamentals of product family architecture". Integrated Manufacturing Systems. Vol. 11, Issue 7, pp. 469-483. 2000. ISSN: 0957-6061. DOI: 10.1108/09576060010349776

[32] B. Agard. "Nomenclature de produits et diversité: étude comparative des approches". Mémoire de DEA, Génie Industriel. Laboratoire GILCO ENSGI-INPG. Grenoble, France. 1999.

[33] T. Simpson, Z. Siddique and J. Jiao. "Product Platform and Product Family Design: Methods and Applications". New York: Springer. 2006. ISBN: 978-1-4614-7937-6.

[34] K. Hölttä-Otto. "Modular Product Platform Design". Doctoral Thesis. Helsinki University of Technology. Espoo, Finland. 2005. ISSN: 1795-4584.

[35] K. Lau Antonio, R.C. Yam and E. Tang. "The impacts of product modularity on competitive capabilities and performance: An empirical study". International Journal of Production Economics. Vol. 105, Issue 1, pp. 1-20. January 2007. ISSN: 0925-5273. DOI:10.1016/j.ijpe.2006.02.002

[36] R. Marshall. "Design modularisation: a systems engineering based methodology for 
enhanced product realisation". Doctoral Theis. Loughborough University. Leicestershire, UK. 1998.

[37] Q. Sun, N. Sridhar and M. O'Brien. "Consumer Perception of Product Stimuli: An Investigation into Indian Consumer Psychology and its Implications for New Product Development, Process and Strategy". D2B2: 2nd Tsinghua International Design Management Symposium. Beijing, China. April 23-26, 2009.

[38] P. Cormier. "Empirically Derived Heuristics to Assist Designers with Satisfying Consumer Variation in Product Design". Proceedings of IDETC/CIE 2011-ASME. International Design Engineering Technical Conferences \& Computers and Information in Engineering. Washington, DC, USA. August 20-31, 2011.

[39] F. Macía S. "Ciencia \& Trabajo". Año 12, N 35, pp. 276-280. Enero/Marzo 2010. ISSN: 0718-0306.

[40] M. Salvador F. y P. Gargallo V. "Análisis factorial". 2012. Fecha de consulta: 3 Diciembre 2012. URL: http://www.5campus. com/leccion/factorial

[41] K.T. Ulrich and K. Tung. "Fundamental of Product Modularity". Working paper $\mathrm{N}^{\circ} 91$ Sloan School of Management. Massachusetts Institute of Technology, ASME. Atlanta, USA. 1991.
[42] M. Crimp. "The Marketing Research Process". Prentice-Hall. New York, USA. 1990. ISBN: 0135577039.

[43] B. Agard. "Contribution a Une Methodologie de Conception de Produits a Forte Diversite". Doctoral Thesis. Institut National Polytechnique de Grenoble. Grenoble, France. 2004.

[44] S. Johansson. "Knowledge, Product Differentiation and Trade". JIBS Dissertation Series $N^{\circ}$ 063. Jönköping International Business School. Jönköping, Sweden. 2010. ISBN: 978-91-86345-07-5.

[45] K. Otto and K. Wood. "Product Design: techniques in reverse engineering and new product development". Prentice Hall. Michigan, USA. 2001. ISBN: 013021271.

[46] P.H. Bloch. "Seeking the ideal form: Product design and consumer response". Journal of Marketing. Vol. 59, Issue 3, pp. 16-29. July, 1995. ISSN: 0022-2429.

[47] S. Lee. "Two Essays on Product Design and Consumer Evaluations". Doctoral Thesis. University of Central Florida. Orlando, USA. 2010.

[48] C.M. Luo and H.F. Chang. "SME competitive strategy: learning from Taiwan's ODM industry". Business Strategy Series. Vol. 12, Issue 3, pp. 107-114. 2011. ISSN: 1751-5637. DOI: $10.1108 / 17515631111130077$. 